\title{
More caries in primary teeth of children who have cleft lip and palate
}

\section{Do children who have cleft lip and palate have elevated caries levels in the primary and permanent dentition?}

\section{Hasslof P, Twetman S.}

Caries prevalence in children with cleft lip and palate - a systematic review of case-control studies. Int J Paediatr Dent 2007; 17:313-319

Data sources Medline (PubMed) was used to identify published data for the review.

Study selection Case-control studies were included if they used controls matched for age and sex, with non-cavitated and manifest caries lesions as outcomes, and were published in English, German, Swedish, Danish or Norwegian.

Data extraction and synthesis The studies were assessed independently by two reviewers and scored A-C according to predetermined criteria for methodology and performance.

Results Significantly more caries was reported in children who had cleft lip and palate (CLP) in two out of the four studies in the permanent dentition and in three out of four publications dealing with primary teeth. None of the articles was scored as ' $A$ ', however, and the level of evidence was therefore based on three papers graded ' $B$ '. There was a tendency towards higher caries scores in preschool children but, as there were conflicting results, the evidence that children with CLP exhibit more caries than noncleft controls was inconclusive.

Conclusions There was a clear tendency towards more caries in primary teeth in children who have CLP. No firm conclusion could be made because the quality of the eligible studies was low to moderate and there are also conflicting results. Better quality research is needed within this area.

\section{Commentary}

The stated aim of this investigation was to examine any evidence of an increased prevalence of dental caries in children who have CLP. The authors wished to confirm or deny the common belief that these children have more untreated caries, especially in the primary dentition. The literature search strategy, exclusion policy and assessment for levels of evidence were clearly described and, of 90 abstracts initially located, 43 papers were assessed by two independent examiners. Six papers ultimately fulfilled the inclusion criteria.

Although significantly more caries was reported in children who had CLP in two out of the four studies in the permanent dentition, and in three out of the four publications dealing with the deciduous teeth; none of these articles was assessed as having the best quality level of evidence. Thus, the authors felt unable to conclude that children with CLP have increased caries prevalence. They noted, however, that none of the studies showed a decreased caries prevalence in the CLP group compared with non-CLP controls and therefore conceded that there may be a tendency towards higher caries scores in preschool children with CLP.

There could be a number of reasons for this. In children who have CLP, aberrant tooth morphology and a collapsed upper dental arch can make plaque removal more difficult. Scarring and a tight upper lip, following primary repair, restrict access to the teeth for both the patient and the dentist. Children with CLP have experienced multiple hospital visits and interventions since infancy, and may view the dentist as a further extension of this, also.

Why is this important? A healthy deciduous dentition is vital for children who have CLP; many of whom will require alveolar bone grafting, preceded by orthodontic expansion, between the ages of 8 and 10 years. Space maintenance and preservation of alveolar bone, especially adjacent to the cleft site, is essential. In the full adult dentition, a healthy mouth is mandatory to allow definitive orthodontics to be carried out.

The conclusion of this well-conducted review was that there is insufficient firm evidence to support the widely held assumption that children with CLP have an increased prevalence of caries. Nevertheless, it is important to recognise that there are certain anatomical and psychological reasons why a CLP may make achieving good oral hygiene and undergoing dental care more challenging.

\section{Julian O'Neill}

Orthodontic Department, Kettering General Hospital NHS Trust, Kettering, Northamptonshire, UK

Evidence-Based Dentistry (2007) 8, 106. doi:10.1038/sj.ebd.6400525

\footnotetext{
Address for correspondence: Svante Twetman, Department of Cariology and Endodontics, School of Dentistry, Faculty of Health Sciences, University of Copenhagen, 20 Nørre Allé, DK-Copenhagen N, Denmark. E-mail: stw@odont.ku.dk
} 